\title{
Society for Social Medicine - fifth annual meeting, 1961
}

(Reprinted from the British Fournal of Preventive $\mathcal{E}$ Social Medicine 1962;16:51)

\begin{abstract}
At a meeting held at the London School of Hygiene and Tropical Medicine on September 28-30, 1961 the proceedings included the presentation of papers of which a selection is abstracted below.
\end{abstract}

\section{Season of birth in relation to other environmental variables \\ I M LECK \\ (Department of Social Medicine, University of Birmingham)}

In the United Kingdom the birth rate fluctuates between a peak in spring and a trough in autumn. From a study of data for England and Wales and for Birmingham, it is concluded that the amplitude of this seasonal fluctuation is relatively low for first births to mothers under 25 and that the time of the seasonal peak may be related to birth order. According to the same data the fluctuation is especially marked for illegitimate births and those of high socio-economic status, but not for multiple births. Analysis of a series of abortions from Belfast suggests that the abortion rate is not increased among winter conceptions. These findings may indicate that the seasonal fluctuation in births is due to variations in human behaviour rather than in the frequency of ovulation or abortion.

\section{Fat in the Rhondda Fach}

GILLIAN R FOWLER

(Introduced by $\operatorname{Dr} R M$ Acheson) (Social and Preventive Medicine Unit, Guy's Hospital

Medical School)

Physique depends upon the relative proportions in the body of muscle, bone, and fat. Fat may be measured with skinfold calipers as the thickness of the subcutaneous layer, a simple and repeatable technique, which taken with height and weight measurements was used in the present study to distinguish the physiques in a random sample of men in two small contrasted occupational groups from the Rhondda Fach. When divided on a two-generation basis into manual labourers, of whom there were 21 , and non-manual workers, of whom there were ten, it was found that the manual labourers had significantly less fat both over the triceps and in the subscapular region.

It is shown that this difference is not due to an age difference between the groups; nor could it be ascribed to the fact that the manual labourers were shorter and lighter, because the ponderal index in the two groups does not differ significantly. Height was found to be independent of fat and of ponderal index, whilst ponderal index is closely related to fat.

For any ponderal index (that is to say, when weight was standardised for height) manual labourers had significantly less fat than the nonmanual workers, so by exclusion the former group must have more muscle and bone. It is thus concluded that the difference between these two groups of men must be due to their body constitutions, rather than to the fact that the non-manual workers have an extra layer of fat over the same underlying amount of muscle and bone.

This suggests an association between physique and occupation, for which one of two explanations is possible: either physique is influenced by occupation, or occupation is influenced by physique. For the former to hold, it must be possible for a man to make considerable changes in both his fat and his muscle mass. No satisfactory evidence could be found that a man can change his muscle mass sufficiently to explain the physical variations observed, so it is contended that these two groups must be of different genotypes and must have selected their jobs because of their physique (consciously or subconsciously) rather than that the jobs are determined by their physique.

\section{Occupational dermatoses in an automobile factory MURIEL NEWHOUSE} (Working with a grant from the MRC, attached to the Institute of Dermatology, St fohn's

Hospital, and the London School of Hygiene and Tropical Medicine)

To assess the prevalence and occupational origin of skin disease, two surveys were undertaken. In the first all patients with skin disease reporting to the factory medical service were interviewed. In the second a random sample of workers in high-risk jobs, chiefly assemblers and machine operatives, were contrasted with workers in low-risk jobs. The second survey revealed a rate almost five times higher than that suggested by the first.

In the second survey the prevalence of dermatitis ranged from 86 per thousand in the assemblers to 18 per thousand in the lowest- 
risk jobs. Folliculitis (a rash directly related to the use of various oils) was chiefly found among the machine operatives and engine assemblers.

Other findings were: that men under 30 were more frequently affected than older men, that men in the first year of service were more frequently affected than in later years, and thirdly that the prevalence among fair-skinned men was significantly greater than among men of dark complexion.

The commonest non-occupational disease was eczema. While the difficulties of distinguishing a constitutional eczema from a dermatitis caused by an injurious substance were stressed, it was pointed out that as a group the eczemas have certain characteristics: the history is longer, the distribution over the body is more widespread, and the personal and familial history of allergy is higher than in the general population.

The final conclusion was that just over half of the skin disease occurring in the factory was of occupational origin.

\section{Maternal anaemia and congenital deformity \\ VICTORIA P COFFEY \\ (Department of Social Medicine, Trinity College, Dublin)}

During a period of 12 months, haemoglobin estimations were done on all women attending the ante-natal clinics of the Coombe Hospital, Dublin, for the first time during their current pregnancy. Those with concentrations of $10 \mathrm{~g}$ per $100 \mathrm{ml}$ or under were regarded as anaemic and the results of their pregnancies were noted carefully. It was not possible to have all the other pregnancies equally carefully observed, but, as controls, careful observations were made on the patients who were next in the series with a haemoglobin over $10 \mathrm{~g}$ per $100 \mathrm{ml}$.

In 94.6 per cent of the anaemic cases the babies were normal and in 5.4 per cent they were stillborn or deformed. The corresponding figures for the controls were 92.4 and 7.6 per cent. respectively. There was, therefore, no significant difference between the two groups.

It was found subsequently that a significantly higher proportion of the patients in the control series had been examined in the first half of pregnancy than of the patients in the anaemic group. It is realised that this may affect the findings materially.

Two other points were noted which are of interest in relation to the high incidence of congenital malformations of the nervous system already noted in this hospital (Coffey and Jessop, 1958[1]):

(a) The proportion of anaemic women whose husbands were in the labouring class or unemployed was 92.3 per cent, compared with 83.3 per cent of the controls, and the proportion of the whole sample in these classes was 88 per cent.

(b) 43 per cent of the anaemic patients had had seven babies or more compared with 25 per cent of the controls.

Coffey P and Jessop W J E (1958). Irish f Med Sci, 6th series.
Effects of weather and air pollution on bronchitic patients

P J LAWTHER, R E WAILER, AND MAUREEN HENDERSON

(MRC Air Pollution Research Unit, St

Bartholomew's Hospital, London)

This paper describes the evolution of a simple technique which has proved valuable in distinguishing between the effects of weather and air pollution upon the health of chronic bronchitic patients. Each patient has recorded his daily state of health in a pocket diary by means of a simple code, and studies amongst groups of patients drawn mainly from chest clinics in the London area have shown that sudden deteriorations in condition are associated with increases in pollution rather than with variations in temperature or humidity. It has been found essential to keep questions as simple as possible and exploratory studies over a period of 5 years have led to two possible approaches in future work. Useful results can be obtained either by using small groups of carefully selected patients who are closely confined in one area or by enrolling large numbers (of the order of 1000) of patients who may then be fairly widely dispersed over an urban area such as Greater London. Routine physiological tests have not been attempted as an integral part of these studies, but parallel work is in progress to determine the nature of the changes in clinical condition during periods of high pollution and to ascertain which pollutant or combination of pollutants may be responsible.

\section{Prematurity-findings in a prospective inquiry \\ ALISON D MCDONALD \\ (Paediatric Research Unit, Guy's Hospital \\ Medical School, London)}

The results of this inquiry are being published elsewhere (Arch Dis Childh and Brit $\mathcal{F}$ Obstet Gynaec).

\section{Arterial pressure, physique and occupation}

C R LOWE

(Depaertment of Social Medicine, Birmingham University)

While employees in a large electrical engineering firm in Birmingham attended a visiting Mass x-Ray Unit, data were collected on arterial pressure, height, weight, arm circumference, and occupation. This paper presents the results of a preliminary analysis of the relation between arterial pressure, physique, and physical demands of occupation for 4780 men aged 20-69 in occupations ranging from management to unskilled serviceman.

At ages above 40 years, both systolic and diastolic pressures were directly related to the physical demands of occupation, the heaviest jobs carrying the highest pressures. It was found, however, that arterial pressure was directly related to weight and to arm circumference and that men doing heavy work weighed more for a given height and had bigger 
arms than men in light or sedentary occupations.

Further analysis is therefore called for to separate these variables.

\section{Spot maps of epidemics}

ALLAN M MCFARLAN

(Department of Public Health, University of

\section{Bristol)}

In studying epidemics of infectious diseases, spot maps are as essential as curves and equations. Early spot maps showed the space distribution of cases in cholera epidemics. It is possible to show both time and space distributions on maps. The methods and results can be illustrated from poliomyelitis epidemics.

Dates can be used instead of dots; different symbols can be used for successive time periods; dots can be marked on a series of outline maps which are drawn on tracing paper and can be superimposed. A regular centrifugal spread or irregularities which demand explanation may be found. Time contours drawn round areas with contemporaneous first cases or epidemic peaks may show centrifugal spread.

Epidemic histograms for parts of an area set beside a diagram of the subdivisions can show geographical spread and differences in the timing of epidemics in the subdivisions estimates of the risk of attack may be wrong if they are calculated on total cases and population when cases are concentrated in one part.

A geographical spread may be due to changes in climatic conditions or in the proportions of susceptibles. MultipIe introductions of infection to an area may obscure the geographical pattern. However, when backed by laboratory and field investigations, the demonstration of geographical spread along the lines of human traffic suggests person-to-person transmission.

\section{Fluctuations in peptic ulcer mortality MERVYN SUSSER}

(Department of Social and Preventive Medicine, University of Manchester)

There has been a decline in mortality from both gastric and duodenal ulcer during the last decade. Signs of a decline can also be found in certain morbidity statistics.
The explanation with the best fit seems to be that the male generations born in the last quarter of the 19th century were exposed, with increasing intensity, to particular environmental hazards which produced a risk of bearing ulcers, and that they have carried this risk throughout life. The wave of gastric ulcers seems to have begun earlier, and receded earlier, than the wave of duodenal ulcers. This cohort hypothesis is supported by an analysis of mortality rates. Although the statistics of the next decade should be awaited to confirm the hypothesis, it seems that some risk particular to the times, apart from the continuing stresses common to industrial societies, should be considered as a contributory cause in ulcer mortality.

\section{Prevalence of mental defect among children in a metropolitan area * J TIZARD AND N GOODMAN (Maudsley Hospital, London)}

The decline during the 20th century in infant mortality, stillbirth rates, and deaths in early life has led to speculation whether the amount of mental defect in the United Kingdom has been increasing.

This paper describes an attempt to measure the prevalence of severe mental defect among children under 16 years of age in Middlesex, and compares the results with those found by Dr E O Lewis in 1925-29.

The prevalence of mongolism had increased four times between the two investigations, and this is in accordance with the findings of Dr C $O$ Carter. The non-mongolian defect, however, was found to have decreased, perhaps by as much as 30 per cent. The possible causes, both methodological and medical or social, include confusion of physical and mental handicap, effect of training, the association between biological and social factors affecting both the mother and the child, and the effects of changes in patterns of fertility.

A plea was made for more information about prevalence in other areas and for conditions other than mongolism.

* An account of this study has now been published elsewhere - Brit Med f 1962;1:216, "Prevalence of Imbecility and Idiocy among Children", by N Goodman and J Tizard. 